Concept Paper

\title{
Correction of Sunglint Effects in High Spatial Resolution Hyperspectral Imagery Using SWIR or NIR Bands and Taking Account of Spectral Variation of Refractive Index of Water
}

\author{
Bo-Cai Gao ${ }^{1,}{ }^{*}$, Rong-Rong Li ${ }^{2}$
}

1. Remote Sensing Division, Code 7232, Naval Research Laboratory, Washington, DC 20375 USA; EMail: gao@nrl.navy.mil

2. Remote Sensing Division, Code 7231, Naval Research Laboratory, Washington, DC 20375 USA; EMail: rong-rong.li@nrl.navy.mil

* Correspondence: Bo-Cai Gao; E-Mail: gao@nrl.navy.mil

Academic Editor: Lei Wang

Special Issue: $\underline{\text { Hyperspectral Remote Sensing }}$

Adv Environ Eng Res

2021, volume 2, issue 3

doi:10.21926/aeer.2103017
Received: April 26, 2021

Accepted: June 15, 2021

Published: July 05, 2021

\begin{abstract}
Passive high spatial resolution hyperspectral and multispectral imaging systems in the solar spectral region from aircraft and satellite platforms are being increasingly used for remote sensing of coastal waters and inland lakes. However, the remotely sensed data are often contaminated by the specular reflection of solar radiation at the air/water interface. Thus, the effects of sunglint need to be corrected. The purpose of sunglint correction is to remove the undesired specular reflected portion of radiance that does not carry any information about the water body and its bottom surface properties and to obtain the water-leaving radiance from the measured total radiance. In general, adequate treatment of the spectral variation of the sunglint effect with due consideration of the physics and the spectroscopy principles has not been done in previously reported hyperspectral sunglint removing techniques. In this study, a practical sunglint removing technique using either a shortwave infrared (SWIR) band
\end{abstract}

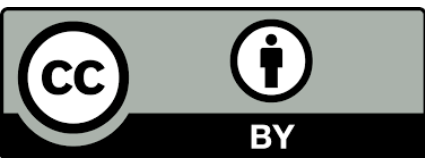

(C) 2021 by the author. This is an open access article distributed under the conditions of the Creative Commons by Attribution License, which permits unrestricted use, distribution, and reproduction in any medium or format, provided the original work is correctly cited. 
or a near-infrared (NIR) band has been developed, where the liquid water absorption is sufficiently large, and the water-leaving radiance (in units of reflectance) is negligible. In the proposed method, proper consideration has been taken for the spectral Fresnel reflection in the wavelength range of $0.35-2.5 \mu \mathrm{m}$. Four cases of sunglint removal from hyperspectral imaging data acquired from two airborne imaging spectrometers have been presented.

\section{Keywords}

Remote sensing; sunglint; ocean color; Fresnel reflection; hyperspectral imaging

\section{Introduction}

Passive high spatial resolution hyperspectral and multispectral imaging systems in the solar spectral region from aircraft and satellite platforms are being increasingly used for remote sensing of coastal waters and inland lakes [1]. In order to use the remotely sensed data to infer about the water bodies and their bottom surface properties, the sunglint effects need to be properly modeled or removed from the remotely sensed data. Figure 1(A) shows an example of a true-color image acquired by the airborne visible infrared imaging spectrometer (AVIRIS) [2] over the land/water boundary area at the Honolulu Airport on April 12, 2000, from an ER-2 aircraft at an altitude of 20 $\mathrm{km}$. Because this dataset was acquired around the local noontime, the surface wave patterns and sunglint effects are present in the image. These effects hinder the capturing of information of the shallow waters and their bottom surface properties. In general, when the downward solar radiance from the sun reaches the waving water surfaces, part of the radiance is secularly reflected at the air/water interface. This reflected portion of radiance is known as the sunglint radiance and does not carry any information about the waters and the underlying surfaces. The other part of the radiance is transmitted into the water, where it is further absorbed and scattered by the constituents within the water body as well as the shallow bottom surface. A minor fraction of the radiance eventually emerges from the water/air interface into the air. The emerging radiance is known as the water-leaving radiance, which carries information about the water body constituents and possibly the bottom surfaces. The sunglint radiance and the water-leaving radiance are then transmitted upwards and reach an aircraft or a satellite sensor. 
(A)

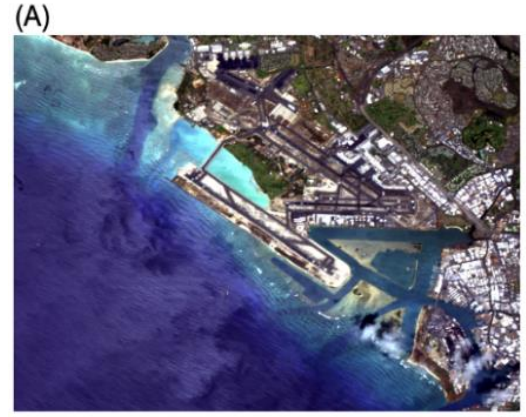

(C)

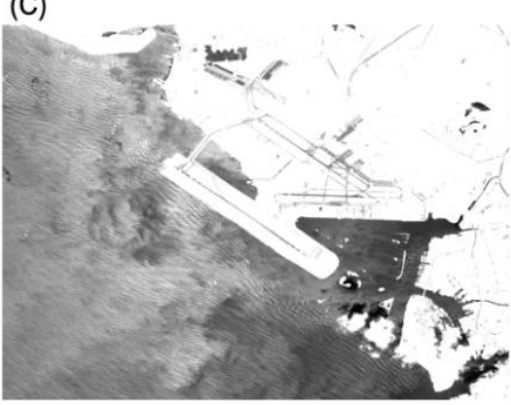

(B)

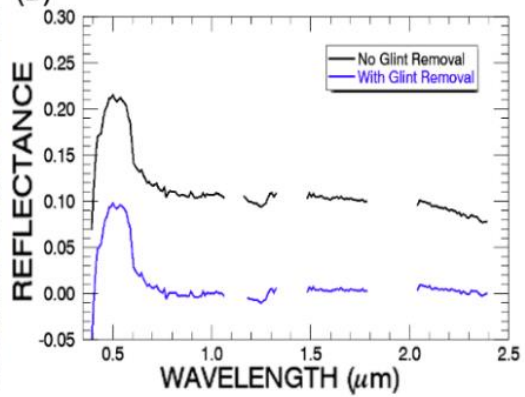

(D)

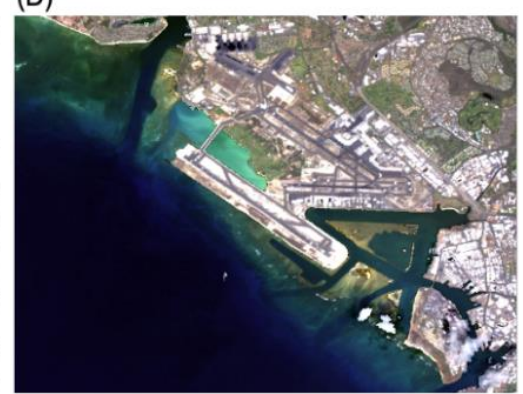

Figure 1 (A) A true-color RGB band image (R: $0.64 \mu \mathrm{m}$; G: $0.55 \mu \mathrm{m} ; \mathrm{B}: 0.46 \mu \mathrm{m}$ ) acquired by AVIRIS over the land/water boundary area at Honolulu Airport on April 12, 2000, from an ER-2 aircraft at an altitude of $20 \mathrm{~km}$, (B) A surface reflectance spectrum of a shallow water pixel after an atmospheric correction (upper curve) and with additional sunglint correction (lower curve), (C) The $1.64 \mu \mathrm{m}$ band image, and (D) the true color RGB band image after atmospheric and sunglint correction. The listed wavelengths are the center positions of the red, green, and blue bands.

There is a long history of developing algorithms for removing the sunglint effect from hyperspectral and multispectral imaging data. As early as 2000, Heege and Fischer [3] described a technique for sun glitter correction in remote sensing imaging spectrometry data. In this technique, the sun glitter correction depends on the information from a band in the shortwave infrared (SWIR) region centered at $1.6 \mu \mathrm{m}$, where no light from the water body itself reaches the sensor because of the strong liquid water absorption at this wavelength. Hochberg et al. [4] described a sunglint correction technique using the correlations between near-infrared (NIR) band images and visible band images. Hedley et al. [5] improved the Hochberg technique and developed a more robust sunglint correction algorithm. Kay et al. [6] presented a thorough review of various sunglint removal algorithms for high as well as low spatial resolution imagery. From the fall of 2009 to the spring of 2010, E. Hochberg led the NASA hyperspectral infrared imager (HyspIRI) sunglint subgroup with the objectives of (1) quantitatively characterizing the sunglint problem, (2) determining the impact of the glint on the aquatic science objectives of the HyspIRI mission, and (3) providing advice for further developments for addressing the glint issue. In 2011, Hochberg et al. produced a comprehensive HyspIRI sunglint subgroup report, which is publicly available online [7]. In this report, we contributed one mitigating method for reducing the effect of sunglint in the visible band images. The method consists of making a nominal hyperspectral atmospheric correction [8, 9], computing the glint reflectance value in the NIR band where the water-leaving reflectance is negligible, and subtracting that value in the visible band images. Subsequently, this method was implemented into a version of an atmospheric correction algorithm for retrieving the water-leaving reflectance from the 
hyperspectral imaging data collected using the portable remote imaging spectrometer (PRISM) [10] during several airborne campaigns. Since 2011, many new developments on sunglint corrections have been reported [11-15]. For example, Zorrilla et al. [11] described an automated SWIR-based empirical sunglint correction method for processing the Landsat 8 operational land imager (OLI) [16] data acquired over turbid coastal waters. Hamel et al. [13] presented a sunglint correction technique for the Sentinel-2 multispectral instrument (MSI) [17] imagery over inland and sea waters using SWIR bands. It should be pointed out that images of different OLI [16] and MSI [17] bands are not acquired simultaneously since water surfaces are nonstationary targets and the wave slope distributions for different bands in a given OLI or an MSI water pixel are not the same. Both Zorrilla et al. [11] and Hamel et al. [13] did not address the issue of wave slope differences explicitly, and thus differences in the sunglint effect in the different OLI and MSI band images were observed. It should be pointed out that the terms NIR and SWIR are frequently used in the ocean color research community, but their wavelength ranges have not been uniquely defined. In this study, NIR light is within the wavelength range of 0.75-1.1 $\mu \mathrm{m}$, whereas SWIR light is within the wavelength range of 1.1-3.0 $\mu \mathrm{m}$.

In all the glint removal studies cited above, the real part of the refractive index of liquid water in the solar spectral region between approximately 0.35 and $2.5 \mu \mathrm{m}$ is assumed to be spectrally flat, i.e., it does not vary with wavelength. Although this is a good first-order assumption, the refractive index of water does decrease slightly with an increasing wavelength of light from the ultraviolet (UV) to the visible region and again in the SWIR region. The assumption of a spectrally flat refractive index of water can result in over- or under-correction to the sunglint effects [7].

In this study, a practical sunglint removal technique using an SWIR band or an NIR band has been developed, where the liquid water absorption is sufficiently strong, and the water-leaving radiance (in units of reflectance) is negligible. Full consideration has been taken for the spectral Fresnel reflection in the wavelength range of $0.35-2.5 \mu \mathrm{m}$. The developed technique was applied for sunglint removal from hyperspectral imaging data acquired from two airborne imaging spectrometers.

The remainder of this paper has been organized as follows: the sunglint correction method is described in Section 2. The sample results obtained by applying the proposed method to several hyperspectral imaging datasets have been presented in Section 3. A brief discussion on the results has been given in Section 4. Finally, a summary of the work is given in Section 5.

\section{Materials and Methods}

\subsection{Absorption and Reflection Properties of Pure Waters}

In order to understand the dependence of the spectral reflectance of sunglint on the wavelength, an understanding of the basic absorption and reflection properties of pure water is necessary. Figure $2(A)$ shows the real part of the refractive index of water $[18,19]$ as a function of the light wavelength. From 0.35 to $0.65 \mu \mathrm{m}$, the refractive index changes from approximately 1.36 to 1.33 . From 0.65 to $2.0 \mu \mathrm{m}$, the refractive index decreases slowly from approximately 1.33 to 1.297 . In the wavelength range of 2.0-2.5 $\mu \mathrm{m}$, the refractive index decreases rapidly with increasing wavelength (from 1.297 to 1.253 ). Figure $2(B)$ shows the inverse of the liquid water absorption coefficient, also known as the "e-folding distance", as a function of wavelength. The "e-folding distance" (in units of a meter) 
is defined as the path length of light of a given wavelength in liquid water over which the light intensity decreases to $e^{-1}$ of the original light intensity. From the curve in Figure 2(B), it can be seen that the e-folding distance is about $1 \mathrm{~m}$ for the light of wavelength $0.7 \mu \mathrm{m}, 0.1 \mathrm{~m}$ at $0.9 \mu \mathrm{m}$, and $0.01 \mathrm{~m}$ at $1.2 \mu \mathrm{m}$. This indicates that light of wavelength longer than approximately $0.9 \mu \mathrm{m}$ would most likely be absorbed completely by liquid water in the ocean after the light entered waters from the air/water interface. The water-leaving radiance/reflectance above $0.9 \mu \mathrm{m}$ should be practically zero, except for the situations where the very top thin layers of ocean waters are quite turbid, or the water surfaces are covered by algae or other floating materials [20].
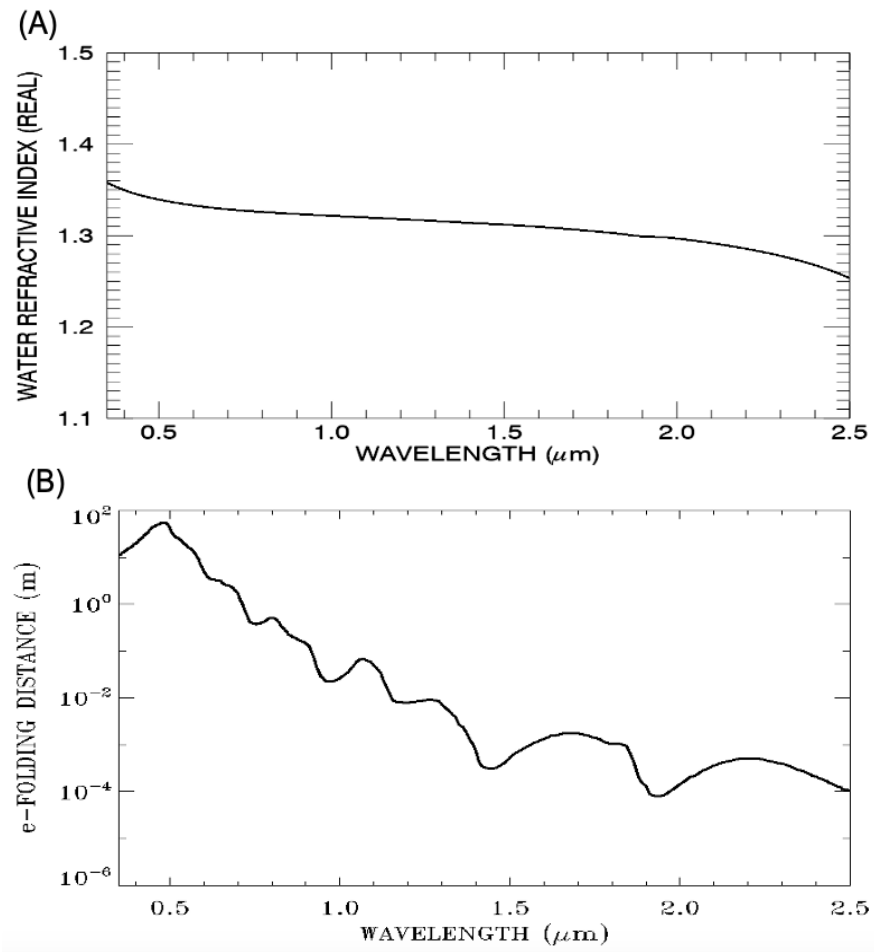

Figure 2 (A) Refractive index of water as a function of wavelength, and (B) the e-folding distance, or the inverse of liquid water absorption coefficient, as a function of wavelength.

\subsection{Fresnel Reflection at the Air/Water Interface}

The waves and sunglint patterns over water surfaces near the Honolulu Airport, as seen in Figure $1(A)$, mainly resulted from the Fresnel reflection of solar radiation at the air/water interface. The well-known Fresnel reflection equation for a horizontally flat air/water interface is

$$
\rho(\theta)=\frac{\left[\frac{\sin ^{2}\left(\theta-\theta^{\prime}\right)}{\sin ^{2}\left(\theta+\theta^{\prime}\right)}+\frac{\tan ^{2}\left(\theta-\theta^{\prime}\right)}{\tan ^{2}\left(\theta+\theta^{\prime}\right)}\right]}{2}
$$

and $\sin \left(\vartheta^{\prime}\right)=\sin (\vartheta) / n$, where $n$ is the real part of the refractive index of water relative to air, $\vartheta$ is the solar zenith angle for the incoming ray in air, $\vartheta^{\prime}$ is the zenith angle for the refracted ray in water, and $\rho(\vartheta)$ is the Fresnel reflectance for unpolarized incoming light. Because of the high spatial resolution of the AVIRIS data with a pixel size of approximately $17 \mathrm{~m}$ at the surface and due to the presence of wave patterns, it is not practically possible to obtain the solar zenith angle relative to 
the local vertical direction of a wave on a pixel-by-pixel basis from the AVIRIS data. Therefore, it is not possible to calculate the Fresnel reflection for each water pixel in the AVIRIS data based on the local solar zenith angle and the real part of the refractive index of water using Eq. (1). In view of this situation, we studied the Fresnel reflection properties for horizontally flat water surfaces but with different incoming solar zenith angles.

Figure $3(\mathrm{~A})$ shows the curves of the Fresnel reflection (from bottom to top) as a function of the light wavelength for solar zenith angles of $0^{\circ}, 15^{\circ}, 30^{\circ}, 45^{\circ}$, and $60^{\circ}$, respectively. The two curves corresponding to the solar zenith angles of $0^{\circ}$ and $15^{\circ}$ almost coincide with each other. Overall, these curves are relatively flat in the $0.7-2.0 \mu \mathrm{m}$ spectral range. Below $0.7 \mu \mathrm{m}$ and above $2 \mu \mathrm{m}$, the reflection values decrease rapidly with increasing wavelength. The shapes of these curves are consistent with the shape of the curve for the real part of the refractive index of water in Figure 2(A).

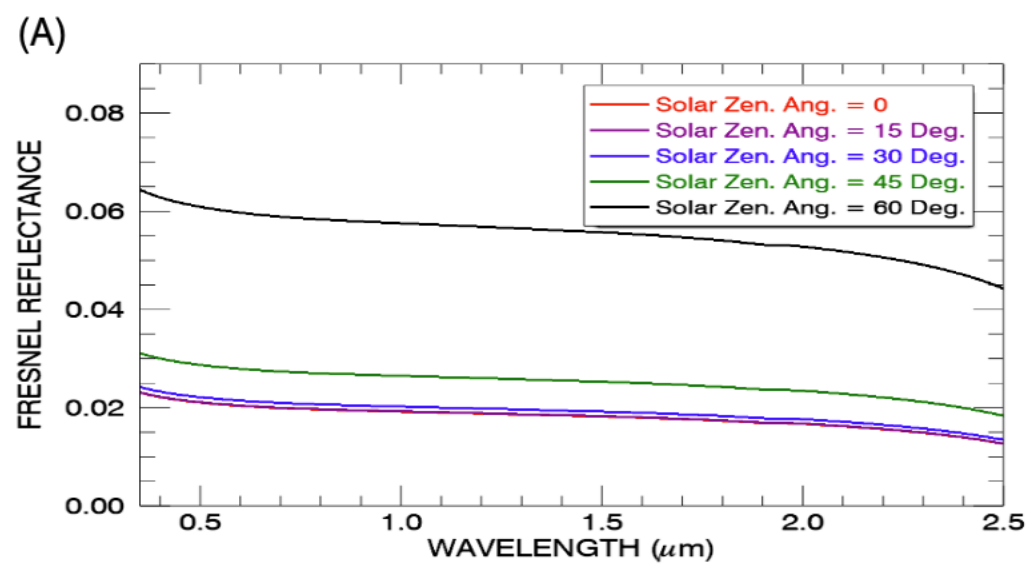

(B)

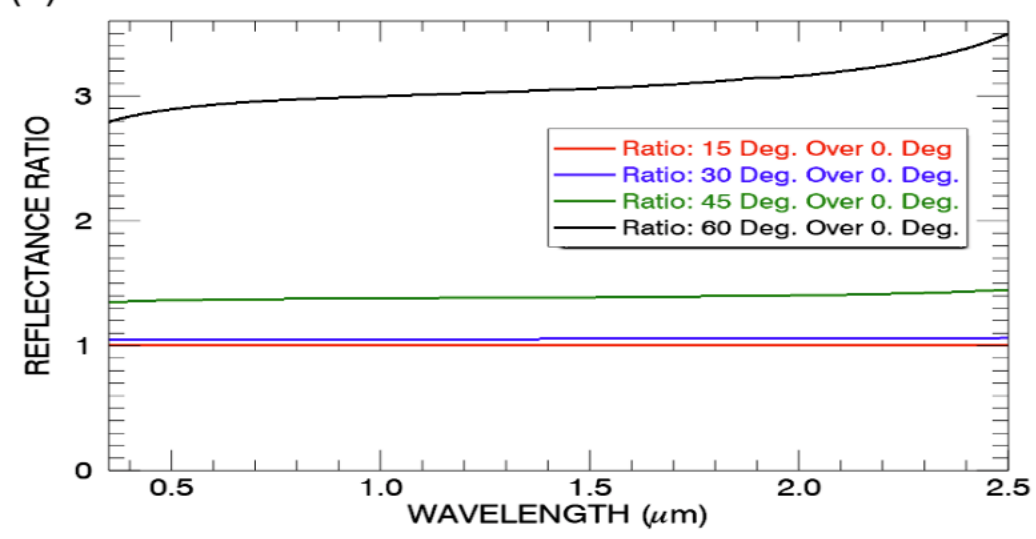

Figure $3(\mathrm{~A})$ Fresnel reflectance as a function of wavelength (from bottom to top) for the solar zenith angles of $0^{\circ}, 15^{\circ}, 30^{\circ}, 45^{\circ}$, and $60^{\circ}$ respectively, and (B) ratio of the reflectance curves (from bottom to top) as a function of wavelength for solar zenith angles of $15^{\circ}, 30^{\circ}, 45^{\circ}$, and $60^{\circ}$ to the reflectance curve for the solar zenith angle of $0^{\circ}$.

Figure 3(B) shows the ratio of the reflectance curves (from bottom to top) as a function of wavelength for the solar zenith angles of $15^{\circ}, 30^{\circ}, 45^{\circ}$, and $60^{\circ}$ to the reflectance curve for the solar zenith angle of $0^{\circ}$. These ratio curves are spectrally flat in the entire wavelength range of $0.35-2.5$ $\mu \mathrm{m}$, except for the ratio curve corresponding to the ratio of the reflectance curve for $60^{\circ}$ solar zenith angle to that for $0^{\circ}$ solar zenith angle. The last curve is not worthwhile for further consideration 
because the reflected solar ray at the large solar zenith angle of $60^{\circ}$ is unlikely to reach an aircraft or a satellite instrument typically with a narrow field of view, and most sunglint effects occur at small solar and view zenith angles. For example, in the case of images acquired by the moderate resolution imaging spectroradiometer (MODIS) [21], the sunglint patterns appear in images with view zenith angles smaller than $40^{\circ}$.

To summarize, the two main points observed from Figures 2(B) and 3(B) are as follows: It can be inferred from Figure 2(B) that, under typical water turbidity conditions, the water-leaving reflectance for bands above $0.9 \mu \mathrm{m}$ is practically zero. From our previous experience of analysis of the AVIRIS data, we have found that the reflectance values for water pixels after removal of the atmospheric and aerosol effects from the hyperspectral imaging data mainly result from sunglint [7]. Let us further assume that the sunglint reflectance value for a given pixel and a given reference

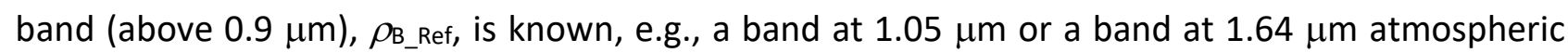
window. We can calculate the ratio, $\mathrm{RTO}_{\mathrm{B}_{-} \mathrm{Ref}}$ of the sunglint reflectance value for this band over the theoretically simulated glint reflectance value for the same brand, but for the zero solar zenith angle condition. Since the bottom three simulated sunglint ratio curves in Figure 3(B) are flat, the shapes of the sunglint reflectance curves in Figure 2(B) are the same as those for solar zenith angles less than approximately $45^{\circ}$. It is only the magnitude of the glint reflectance that depends on the solar zenith angle. At this stage, if we scale all the data points in the theoretically simulated glint reflectance curve for the zero solar zenith condition by the same factor, $\mathrm{RTO}_{\mathrm{B}_{-} \mathrm{Ref}}$, we can derive the spectral glint reflectance curve for a given pixel. The subsequent subtraction of the glint reflectance curve from the atmosphere-corrected reflectance curve would result in the water-leaving reflectance curve for the pixel. These points are further illustrated below.

\subsection{An Illustration of Spectral Sunglint Removal}

The top curve in Figure $1(B)$ is a surface reflectance spectral curve obtained after atmospheric correction, performed using a hyperspectral atmospheric correction algorithm [8], of a shallow water pixel having significant sunglint effects, as observed in the image shown in Figure 1(A). The spectral reflectance values in the spectral range of $0.75-2 \mu \mathrm{m}$ are fairly constant because the refractive index of water (real part) decreases very slowly with increasing wavelength in this spectral range (see Figure 2(A)). These reflectances are mainly the results of sunglint. Above $2 \mu \mathrm{m}$, the reflectance values decrease with increasing wavelength due to the rapid decrease in the refractive index of water (also see Figure 2(A)) with increasing wavelength in this spectral interval. Thus, a reference band centered near $1.64 \mu \mathrm{m}$ was selected, and a sunglint reflectance value, $\rho_{B_{-} \text {Ref, of } 0.104}$ was obtained from this spectrum. The simulated Fresnel reflection value, $\rho_{B_{-} \text {Simu, }}$ for this reference

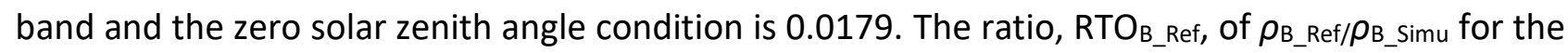
$1.64 \mu \mathrm{m}$ reference band was calculated and multiplied to all the data points in the simulated glint spectrum corresponding to the zero solar zenith angle condition. Subsequently, a complete sunglint spectrum for this particular water pixel was derived. Finally, the glint spectrum was subtracted from the atmosphere-corrected spectrum and the sunglint removed water-leaving reflectance spectrum was obtained, which is shown by the blue-colored line in Figure 1(B). The water-leaving reflectance spectrum is fairly flat in the spectral range of $0.75-2.5 \mu \mathrm{m}$. The small variations in this wavelength range are due to our inability to model all the fine atmospheric gas absorption features when performing atmospheric corrections and due to the limited signal-to-noise ratio of the AVIRIS data. 
A fairly large reflectance bump is observed in the derived water-leaving reflectance spectrum in the visible region with a peak reflectance value of approximately 0.1 . Below $\sim 0.4 \mu \mathrm{m}$, the water-leaving reflectance values become negative. This is related to the radiometric calibration errors (of the order of 20\%) of the AVIRIS data in the UV and blue spectral regions. Figure 4 shows a flowchart illustrating the procedure for removing spectral sunglint effects from a water pixel.

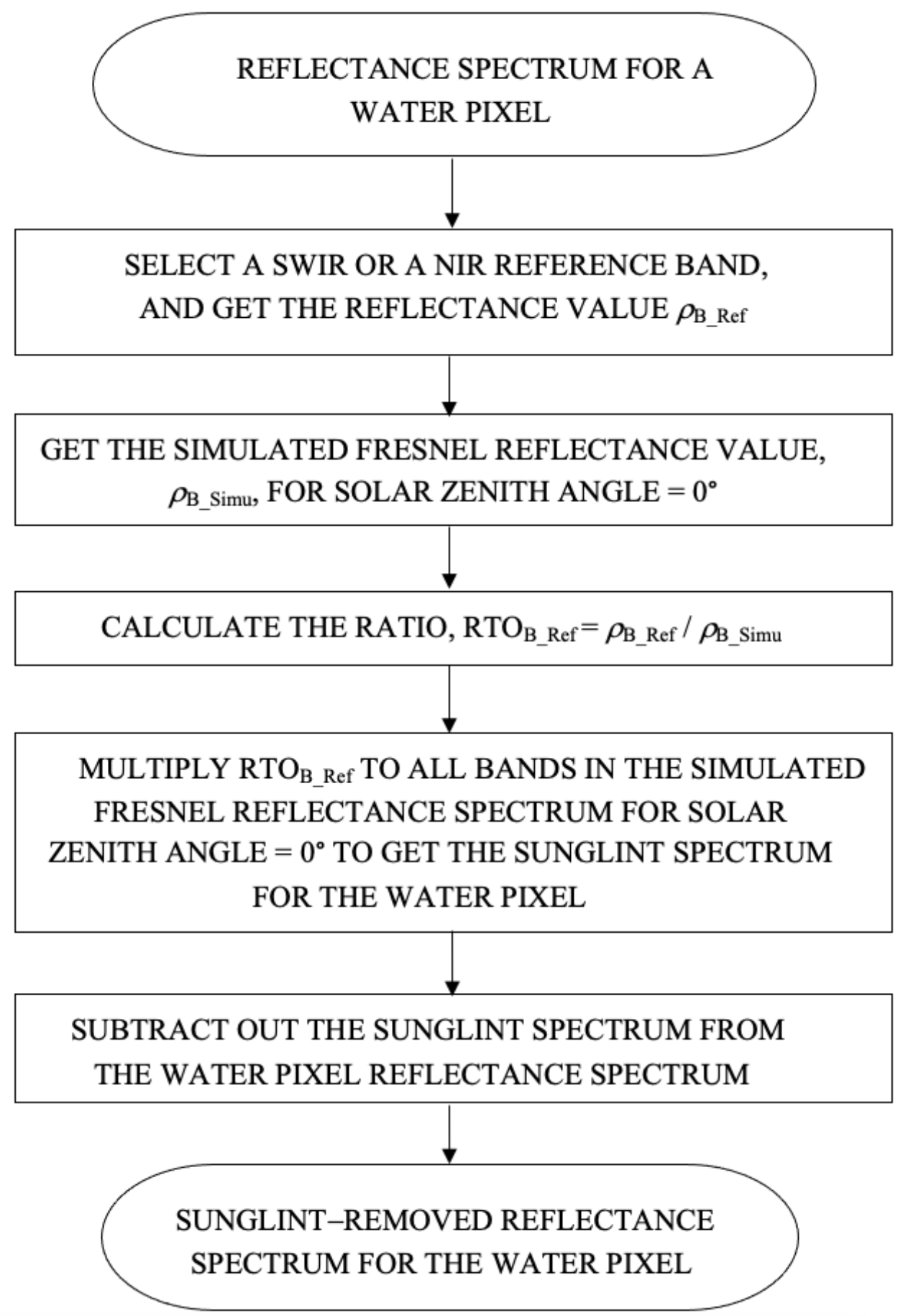

Figure 4 Flowchart illustrating the steps for removing the spectral sunglint effects for a water pixel.

\section{Results}


We selected four cases to demonstrate our technique for removing the sunglint effect from images. Out of the four, three cases were selected from the large volume of the AVIRIS dataset collected in the spring of 2000 during a NASA-sponsored airborne campaign conducted over the Hawaii water bodies. At the time, no special planning for the high-altitude ER-2 flight time and flight directions was made to minimize the sunglint in order to survey as large an area as possible. As a result, many images captured by AVIRIS were affected by sunglint. The last case was a PRISM dataset collected in 2016 over a coral reef site near Lizard Island in the Pacific Ocean during the NASA sponsored coral reef airborne laboratory (CORAL) [22] field campaign led by E. Hochberg of the Bermuda Institute of Ocean Sciences.

\subsection{Image from AVIRIS of the Honolulu Airport Area, April 12, 2000}

The first selected case is an image acquired by AVIRIS over the land/water boundary area at the Honolulu Airport on April 12, 2000, from an ER-2 aircraft at an altitude of $20 \mathrm{~km}$. As described in the previous section, the RGB band image (Red: $0.64 \mu \mathrm{m}$, Green: $0.55 \mu \mathrm{m}$, Blue: $0.46 \mu \mathrm{m}$ ) is shown in Figure $1(A)$. An example of the retrieved surface reflectance spectrum and the subsequent sunglint removal is illustrated in Figure $1(B)$, whereas Figure $1(C)$ shows the $1.64 \mu \mathrm{m}$ band image. In order to reveal the features of the water body and its possible underneath surface, the image in Figure 1 (C) was stretched to saturate the land pixels. Even in such a highly stretched image, no water and bottom surface features are observed in it, thus indicating that there is almost no water-leaving reflectance over the water pixels in this image. Only the waves and glint patterns are observed. Figure $1(D)$ shows the RGB band image after atmospheric and sunglint corrections. From a comparison of this image with that shown in Figure $1(A)$, it can be seen that the surface wave patterns are successfully removed, and the spatially contiguous water body and bottom surface features are revealed quite well. In order to avoid making sunglint corrections for the land pixels in the image shown in Figure 1(D), a simple land/water mask was used. This mask is largely based on the normalized difference between one band at $0.86 \mu \mathrm{m}$ and another band at $0.65 \mu \mathrm{m},[\rho(0.86 \mu \mathrm{m})$ $-\rho(0.65 \mu \mathrm{m})] /[\rho(0.86 \mu \mathrm{m})+\rho(0.65 \mu \mathrm{m})]$, in the spectrum corrected of the atmospheric effects (e.g., the upper curve in Figure $1(\mathrm{~B}))$. In general, the normalized difference is negative for a water pixel and positive for a land pixel, and the exceptions are rare.

\subsection{Image from AVIRIS Over the French Frigate Shoals, April 18, 2000}

The second selected case is an image acquired by AVIRIS over the French Frigate Shoals area, $23.62^{\circ} \mathrm{N}$ and $166.1^{\circ} \mathrm{W}$ in the Pacific Ocean, on April 18, 2000. Figure 5(A) shows the RGB band image in which the waves and sunglint features can be observed in the right portion of the scene. Figure 5(B) shows the $1.64 \mu \mathrm{m}$ band image, where the waves and sunglint patterns are seen. However, the underwater atoll features in the lower left portion of Figure $5(A)$ are completely absent in the image shown in Figure 5(B). This demonstrates that the water-leaving reflectance is close to zero in the $1.64 \mu \mathrm{m}$ band image. Figure 5(C) shows the RBG image after atmospheric and sunglint corrections. By comparing Figure $5(C)$ with $5(A)$, it can be seen that the wave and glint patterns are properly removed, and the underwater atoll features are properly recovered in the image shown in Figure 5(C). 


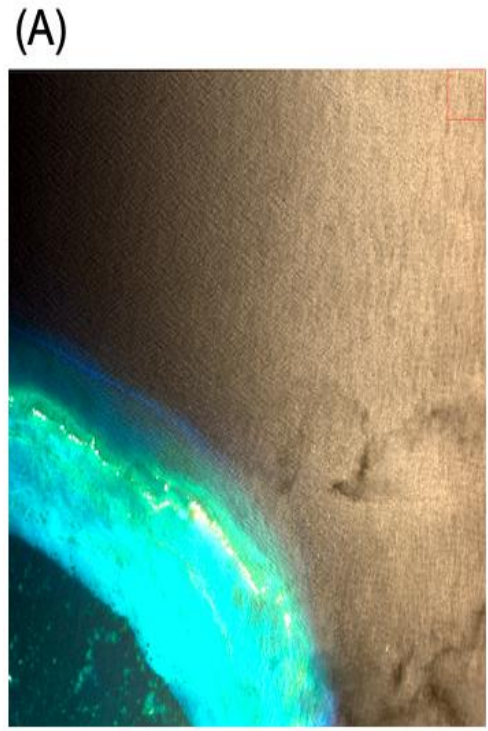

(B)

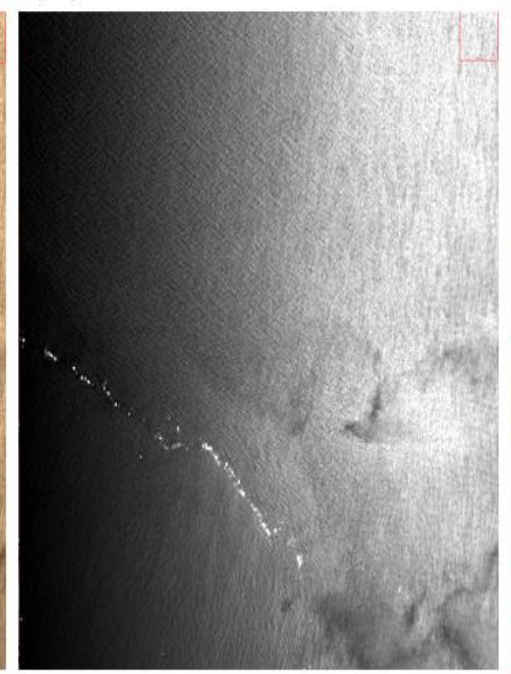

(C)

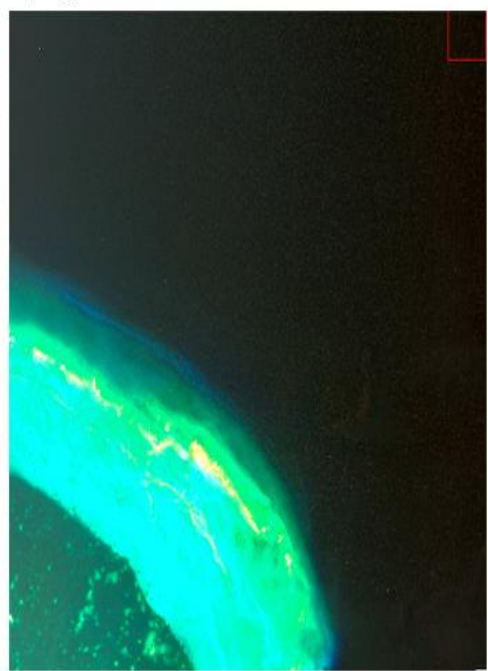

Figure 5. (A) A true-color RGB band image (R: $0.64 \mu \mathrm{m}, \mathrm{G}: 0.55 \mu \mathrm{m}, \mathrm{B}: 0.46 \mu \mathrm{m}$ ) acquired by AVIRIS over the French Frigate Shoals area in the Pacific Ocean on April 18, 2000, from an ER-2 aircraft at an altitude of $20 \mathrm{~km}$, (B) shows the $1.64 \mu \mathrm{m}$ band image of the same scene, and (C) shows the true-color RGB band image after atmospheric and sunglint corrections.

\subsection{Image from AVIRIS of Kaneohe Bay, April 12, 2000}

The third case presented here is an image acquired by AVIRIS on April 12, 2000, of the Kaneohe Bay, centered near $21.46^{\circ} \mathrm{N}$ and $157.81^{\circ} \mathrm{W}$. It is located in the northeast coastal area of $\mathrm{O}^{\prime}$ ahu Island in Hawaii. The bay contains the largest sheltered body of water among all the Hawaiian Islands. Figure 6(A) shows the RGB band image of Kaneohe Bay in which the wave patterns and specular reflection effects are present over the water body. Figure 6(B) shows the corresponding RGB band image after atmospheric and sunglint corrections. The underwater corals and bright sandy bars in the bay can be clearly seen after the corrections. Figure 6(C) shows an example of the surface reflectance spectrum for a water pixel after atmospheric correction (the upper black curve) and after sunglint correction (the lower blue curve), which accounts for the effect of the spectral variation of the refractive index of water with wavelength. 

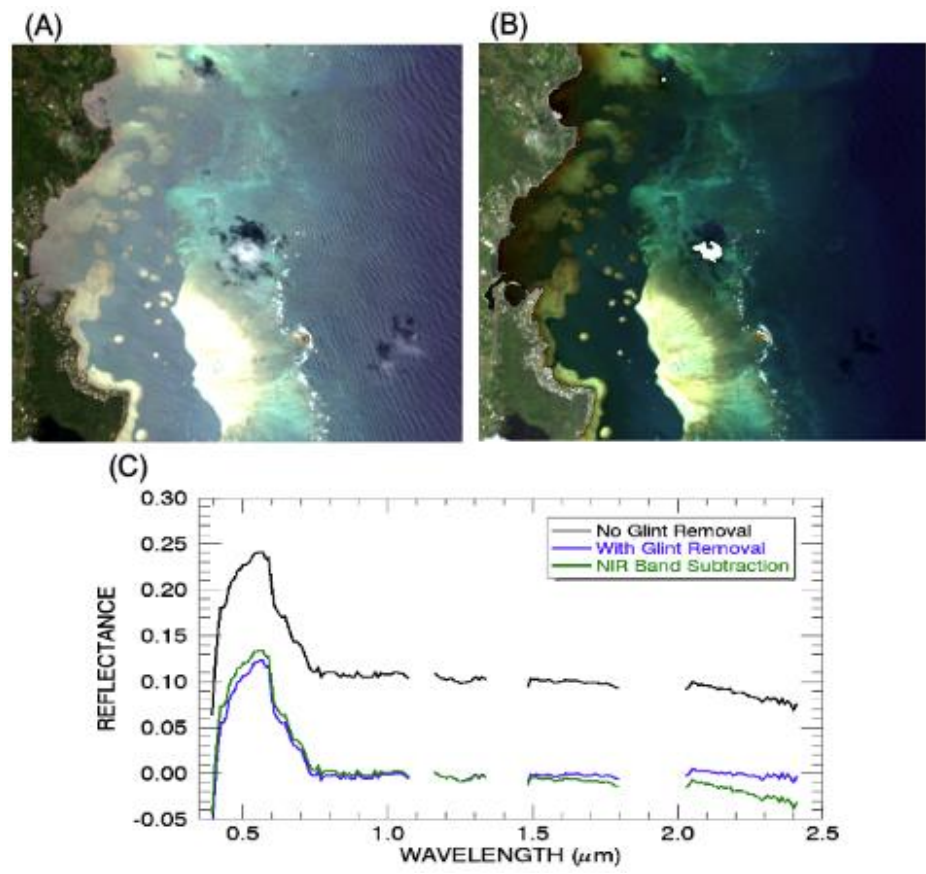

Figure 6 (A) A true-color RGB band image (R: $0.64 \mu \mathrm{m}, \mathrm{G}: 0.55 \mu \mathrm{m}, \mathrm{B}: 0.46 \mu \mathrm{m}$ ) acquired by AVIRIS over Kaneohe Bay located in the northeastern coastal area of $O$ 'aho Island in Hawaii, (B) shows the corresponding RGB band image after atmospheric and sunglint corrections, and (C) shows the surface reflectance spectrum of a water pixel after atmospheric correction (upper black curve), after sunglint correction done by taking the spectral variation of the real part of the refractive index of water into consideration (lower blue curve), and after sunglint correction done using the simple NIR subtraction method [7] (lower green curve).

For comparison, sunglint corrections have also been made using the simple NIR reflectance subtraction technique [7] and using the technique reported by Goodman et al. [23]. Both techniques have been previously used by the ocean color research community. In our implementation of the NIR subtraction technique, the NIR band centered at $0.865 \mu \mathrm{m}$ was selected as the reference band. The reflectance value for this band after atmospheric correction (see the top black curve in Figure $6(C)$ was subtracted from all the data points in the black curve. The resulting sunglint-corrected water-leaving reflectance spectrum is shown as the green-colored curve in Figure 6(C). The green curve exhibits a downward trend in the spectral range of 1.5-2.5 $\mu \mathrm{m}$, whereas the blue curve is fairly flat and clustered around a horizontal line with a zero reflectance value. Therefore, the spectral variation of the refractive index of water as a function of wavelength needs to be taken into account while performing the sunglint correction in order to properly recover the water-leaving reflectance in the SWIR region. The simple NIR subtraction method obviously over-corrects the sunglint effect above $2 \mu \mathrm{m}$. In particular, in the $2.25 \mu \mathrm{m}$ narrow 'atmospheric window' region, the over-correction is 0.023 in units of reflectance. The difference between the green and blue curves at $0.55 \mu \mathrm{m}$ is relatively smaller (0.012 in units of reflectance). For making further comparisons, the sunglint corrections were also made using the method developed by Goodman et al. [23]. There are two reference bands involved with this method, one located at $0.64 \mu \mathrm{m}$ and the other at $0.75 \mu \mathrm{m}$. The resulting sunglint-corrected spectrum (not shown in Figure 6(C)) is almost identical to the greencolored spectrum obtained using the simple NIR reflectance subtraction method. In order to access 
the effects of the different sunglint removal algorithms on the geophysical parameter retrievals, chlorophyll concentrations were derived from our sunglint-corrected spectra and spectra obtained using the NIR subtraction method. Noticeable differences in the sunglint-corrected spectra in the spectral range of 0.45-0.7 $\mu \mathrm{m}$ could be seen, as shown by the blue and green curves in Figure $6(C)$. A band ratio version of the NOAA algorithm $(0.491 \mu \mathrm{m} / 0.554 \mu \mathrm{m})$ was used for chlorophyll retrievals. The mean difference in the chlorophyll concentrations derived from the two sunglintcorrected datasets was observed to be relatively small ( 11\%). This is because the chlorophyll algorithm depends on the ratio of the two bands and the absolute reflectance values of the two bands are less important after the ratio calculation.

\subsection{Image from PRISM Obtained Near the Lizard Island in the Pacific Ocean, October 14, 2016}

The fourth case is an image acquired by PRISM very close to the Lizard Island in the Pacific Ocean on October 14, 2016, during the NASA sponsored CORAL field campaign. The image coordinates are centered near $13.90^{\circ} \mathrm{S}$ and $146.63^{\circ} \mathrm{W}$. The Lizard Island itself is an island on the Great Barrier Reef in Queensland, Australia. Compared to AVIRIS, PRISM provides an improved signal-to-noise ratio, finer spectral sampling interval, and higher spectral resolution $[2,10]$. However, PRISM covers a smaller wavelength range between $0.35-1.05 \mu \mathrm{m}$.

Figure $7(A)$ shows the RGB band image, which was acquired exclusively over a water body that does not have any solid land coverages. The right portion of the image was affected by sunglint. Because the scene had no land coverage, no land/water mask was required when performing the glint correction. Figure 7(B) shows the RGB band image after atmospheric and sunglint corrections. The upper curve in Figure $7(C)$ corresponds to a surface reflectance spectrum obtained after atmospheric corrections for a shallow water pixel having a reflection from the bottom surface. The spectral glint reflectance in the 0.75-1.05 $\mu \mathrm{m}$ range is fairly flat. During the sunglint correction process, an NIR band centered at $0.86 \mu \mathrm{m}$ was selected as the reference band for computing the sunglint reflectance value, $\rho_{B_{-} \text {Ref. }}$ The lower curve (light blue colored curve) in Figure $7(\mathrm{C})$ is the water-leaving reflectance spectrum obtained after sunglint correction.
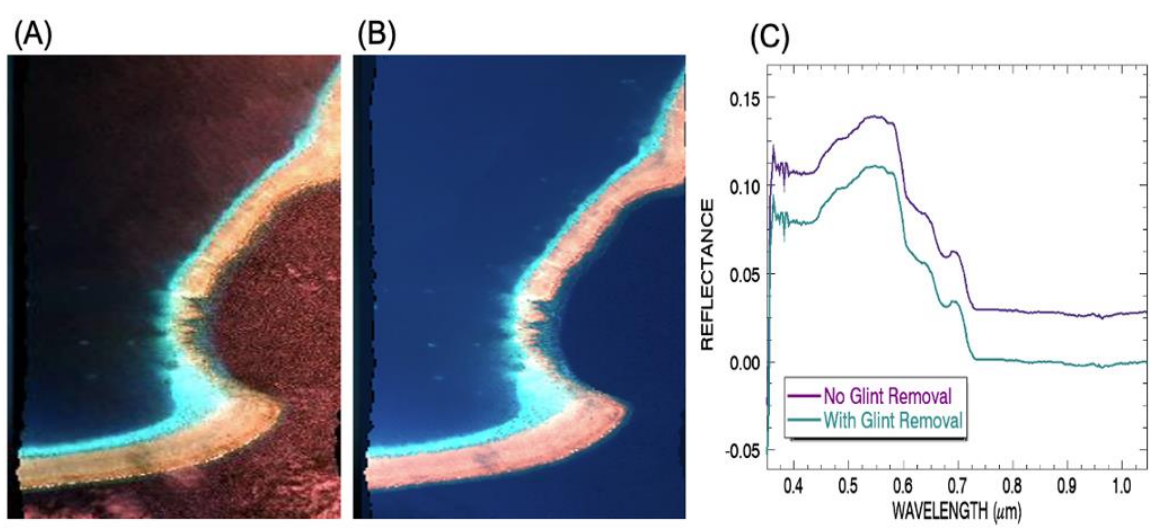

Figure 7. (A) A true-color RGB band image (R: $0.64 \mu \mathrm{m}, \mathrm{G}: 0.55 \mu \mathrm{m}, \mathrm{B}: 0.46 \mu \mathrm{m}$ ) acquired using PRISM near the Lizard Island in the Pacific Ocean, (B) shows the corresponding RGB band image obtained after atmospheric and sunglint corrections, and (C) shows the surface reflectance spectrum of a water pixel obtained after atmospheric correction (upper curve) and sunglint correction (lower curve). 
It should be pointed out that the surface reflectance spectrum without glint correction, such as the upper curve in Figure $7(C)$, cannot be directly used for deriving the properties of the water body and its bottom surface using the underwater radiative transfer models and retrieving algorithms [24, 25]. This is because the algorithms would be unable to simulate the large reflectance values above approximately $0.75 \mu \mathrm{m}$. On the other hand, the glint-corrected water-leaving reflectance spectrum, such as the lower curve in Figure $7(C)$, is suited for retrieving the water body and its bottom surface properties using the underwater radiative transfer models and retrieving algorithms [26].

\section{Discussion}

In this study, four cases were discussed in which sunglint removal was performed using our developed method. For the first three cases, images from the AVIRIS dataset covering the solar spectral range of 0.4-2.5 $\mu \mathrm{m}$ were used. A SWIR band centered near $1.64 \mu \mathrm{m}$ was selected as the reference band for performing the sunglint correction. In principle, a SWIR band centered near 2.2 $\mu \mathrm{m}$ can also be selected as the reference band. However, the band near $2.2 \mu \mathrm{m}$ in the AVIRIS dataset is much noisier than the $1.64 \mu \mathrm{m}$ band. In order to avoid the possible noise propagation to the sunglint-removed bands in the visible region, instead of the $2.20 \mu \mathrm{m}$ SWIR band, the $1.64 \mu \mathrm{m}$ SWIR band was selected as the reference band. For the fourth case, an image from the PRISM dataset was selected. Because the PRISM instrument does not cover the 1.05-2.5 $\mu \mathrm{m}$ SWIR spectral range, a PRISM NIR band centered at $0.86 \mu \mathrm{m}$ was selected as the reference band. Overall, in our sunglint removing method, only one reference band, either a SWIR band or an NIR band, was required. After considering the spectral coverage and the instrument noise characteristics, a reference band at the longer wavelength end was chosen because of the smaller water-leaving reflectance for the band.

The refractive index of water reported by Segelstein [18] was used in this study. The same set of refractive index values has been used extensively in the atmospheric sciences community for remote sensing of water clouds [21]. The refractive index of water is, in practice, a function of the temperature and salinity of water $[19,27,28]$. As the temperature and salinity change, the water density changes slightly, and therefore the real part of the refractive index of water also changes slightly. On the other hand, the trend of the real part of the refractive index of water as a function of wavelength remains the same. In our formulation of the sunglint removing algorithm, as described in Section 2, only the shapes of the curves of the simulated Fresnel reflection as a function of wavelength were used; their absolute reflectance values were not used. As a result, if a different real part of the refractive index table of water was used in our simulation, the newly obtained spectral sunglint reflectance curve for a given pixel would, in principle, remain approximately the same.

In the late 1980s, a hyperspectral imager was required to have the ability to measure all bands within a given pixel simultaneously [29]. Our technique described in this article is applicable for removing sunglint effects from this type of hyperspectral dataset, because the movement of the wave facets between the data acquisition for different bands is not an issue. However, there are different types of hyperspectral imagers that are used for acquiring three-dimensional data cubes (two dimensions in the spatial domain and one dimension in the spectral domain). For example, a hyperspectral imager based on the wedge filter technology [30] does not capture different bands in a spectrum over a given pixel simultaneously. The movement of the wave facets between data acquisitions for different bands can result in changes in the magnitudes of Fresnel reflection. The 
multichannel Landsat8 OLI instrument [16] and the Sentinel2 MSI instrument [17] also do not acquire images of different bands simultaneously and have the same wave facet movement issue among different band images.

\section{Conclusions}

We have described a practical sunglint removing method using a SWIR band or an NIR band, where the liquid water absorption coefficient is sufficiently large, and the water-leaving radiance (in the units of reflectance) is negligible. Careful consideration was taken of the shapes of the spectral Fresnel reflection as a function of the wavelength of light in the range of $0.35-2.5 \mu \mathrm{m}$. The technique developed in this study for sunglint removals was applied to the hyperspectral imaging data acquired from two airborne imaging spectrometers. Our method is most applicable to hyperspectral imaging data acquired over water surfaces in which all the bands within a pixel are acquired simultaneously. We do not recommend the use of this method or the concept presented in this article for sunglint removal from hyperspectral imaging data or multispectral imaging datasets, where the data for different bands in a given pixel are not acquired simultaneously. This is because the movement of the wave facets between data acquisitions for different bands can result in changes in the magnitude of Fresnel reflection for the bands.

\section{Acknowledgments}

The authors are grateful to Eric Hochberg with Bermuda Institute of Ocean Sciences for many useful discussions.

\section{Author Contributions}

BCG made initial algorithm development. RRL carried out retrievals and detailed data analysis.

\section{Funding}

This research is partially supported by the US Office of Naval Research and by a research grant managed by Jared K. Entin at the Science Mission Directorate of National Aeronautics and Space Administration.

\section{Competing Interests}

The authors declare no conflict of interest.

\section{References}

1. Kudela RM, Palacios SL, Austerberry DC, Accorsi EK, Guild LS, Torres-Perez J. Application of hyperspectral remote sensing to cyanobacterial blooms in inland waters. Remote Sens Environ. 2015; 167: 196-205.

2. Green RO, Eastwood ML, Sarture CM, Chrien TG, Aronsson M, Chippendale BJ, et al. Imaging spectrometry and the airborne visible/infrared imaging spectrometer (AVIRIS). Remote Sens Environ. 1998; 65: 227-248. 
3. Heege $T$, Fischer J. Sun glitter correction in remote sensing imaging spectrometry. Proceedings of the SPIE Ocean Optics XV Conference; 2000 October 16-20; Monaco.

4. Hochberg E, Andrefouet S, Tyler MR. Sea surface correction of high spatial resolution Ikonos images to improve mapping in near-shore environments. IEEE Trans Geosc Remote Sens. 2003; 41: 1724-1729.

5. Hedley JD, Harborne AR, Mumby PJ. Simple and robust removal of sun glint for mapping shallow-water benthos. Int J Remote Sens. 2005; 26: 2107-2112.

6. Kay S, Hedley JD, Lavender S. Sun glint correction of high and low spatial resolution images of aquatic scenes: A review of methods for visible and near-infrared wavelengths. Remote Sens. 2009; 1: 697-730.

7. Hochberg EJ, Mobley C, Park Y, Goodman J, Turpie KR, Gao BC, et al. Summary of HyspIRI sunglint subgroup dscussions and analyses Fall 2009 - Spring 2010 [Internet]. Available from: https://www.researchgate.net/publication/259827816 Summary of HyspIRI Sunglint Subgr oup Discussions and Analyses Fall 2009 - Spring 2010\#fullTextFileContent.

8. Gao BC, Heidebrecht KB, Goetz AF. Derivation of scaled surface reflectances from AVIRIS data. Remote Sens Environ. 1993; 44: 165-178.

9. Gao BC, Montes MJ, Ahmad Z, David CO. Atmospheric correction algorithm for hyperspectral remote sensing of ocean color from space. Appl Opt. 2000; 39: 887-896.

10. Mouroulis P, Van Gorp B, Green RO, Dierssen H, Wilson DW, Eastwood M, et al. Portable remote imaging spectrometer coastal ocean sensor: Design, characteristics, and first flight results. Appl Opt. 2014; 53: 1363-1380.

11. Zorrilla NA, Vantrepotte V, Ngoc DD, Hyybrechts N, Gardel A. Automated SWIR based empirical sun glint correction of Landsat 8-OLI data over coastal turbid water. Opt Express. 2019; 27: A294-A318.

12. Chen J, He XQ, Liu ZL, Lin N, Xing QG, Pan DL. Sun glint correction with an inherent optical properties data processing system. Int J Remote Sens. 2021; 42: 617-638.

13. Harmel T, Chami M, Tormos T, Reynaud N, Danis PA. Sunglint correction of the Multi-Spectral Instrument (MSI)-SENTINEL-2 imagery over inland and sea waters from SWIR bands. Remote Sens Environ. 2018; 204: 308-321.

14. Duan PH, Lai JB, Kang J, Kang XD, Ghamisi P, Li ST. Texture-aware total variation-based removal of sun glint in hyperspectral images. ISPRS J Photogramm Remote Sens. 2020; 166: 359-372.

15. Vanhellemont $Q$. Adaptation of the dark spectrum fitting atmospheric correction for aquatic applications of the Landsat and Sentinel-2 archives. Remote Sens Environ. 2019; 225: 175-192.

16. Storey J, Choate M, Lee K. Landsat 8 operational land imager on-orbit geometric calibration and performance. Remote Sens. 2014; 6: 11127-11152.

17. Drusch M, Del Bello U, Carlier S, Colin O, Fernandez V, Gascon F, et al. Sentinel-2: ESA's optical high-resolution mission for GMES operational services. Remote Sens Environ. 2012; 120: 25-36.

18. Segelstein DJ. The complex refractive index of water. Kansas City, MO: University of Missouri; 1981.

19. Kou L, Labrie D, Chylek P. Refractive indices of water and ice in the 0.65 - to $2.5-\mu \mathrm{m}$ spectral range. Appl Opt. 1993; 32: 3531-3540.

20. Gao BC, Li RR. FVI - a floating vegetation index formed with three near-IR channels in the 1.0 $1.24 \mu \mathrm{m}$ spectral range for the detection of vegetation floating over water surfaces. Remote Sens. 2018; 10: 1421. 
21. King MD, Menzel WP, Kaufman YJ, Tanre D, Gao BC, Platnick S, et al. Cloud and aerosol properties, precipitable water, and profiles of temperature and humidity from MODIS. IEEE Trans Geosci Remote Sens. 2003; 41: 442-458.

22. About CORAL [Internet]. Jet Propulsion Laboratory. Available from: https://coral.jpl.nasa.gov/about-coral.

23. Goodman JA, Lee ZP, Ustin SL. Influence of atmospheric and sea-surface corrections on retrieval of bottom depth and reflectance using a semi-analytical model: A case study in Kaneohe Bay, Hawaii. Appl Opt. 2008; 47: F1-F11.

24. Lee ZP, Carder KL, Mobley CD, Steward RG, Patch JS. Hyperspectral remote sensing for shallow waters. 1. A semianalytical model. Appl Opt. 1998; 37: 6329-6338.

25. Lee ZP, Carder KL, Mobley CD, Steward RG, Patch JS. Hyperspectral remote sensing for shallow waters. 2. Deriving bottom depths and water properties by optimization. Appl Opt. 1999; 38: 3831-3843.

26. Garcia RA, Lee ZP, Hochberg EJ. Hyperspectral shallow-water remote sensing with an enhanced benthic classifier. Remote Sens. 2018; 10: 147.

27. Daimon M, Masumura A. Measurement of the refractive index of distilled water from the nearinfrared region to the ultraviolet region. Appl Opt. 2007; 46: 3811-3820.

28. Aly KM, Esmail E. Refractive index of salt water: Effect of temperature. Opt Mater. 1993; 2: 195199.

29. Goetz AF, Herring M. The high resolution imaging spectrometer (HIRIS) for Eos. IEEE Trans GeosC Remote Sens. 1989; 27: 136-144.

30. Ungar SG, Pearlman JS, Mendenhall JA, Reuter D. Overview of the earth observing one (EO-1) mission. IEEE Trans Geosc Remote Sens. 2003; 41: 1149-1159.

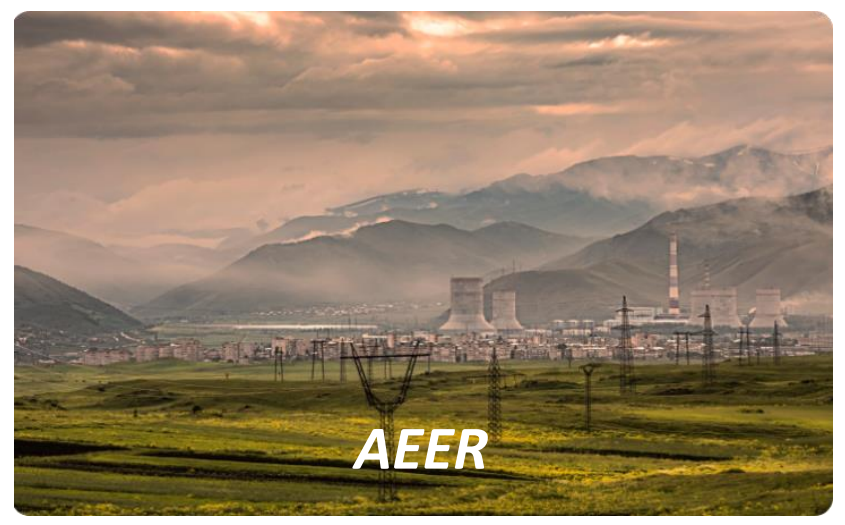

Enjoy AEER by:

1. Submitting a manuscript

2. Joining in volunteer reviewer bank

3. Joining Editorial Board

4. Guest editing a special issue

For more details, please visit: http://www.lidsen.com/journals/aeer 